\title{
Sprach- und kulturbedingte Mißverständnisse in deutsch-ungarischen Geschäftsbeziehungen
}

\author{
Dr. Tiborc Fazekas
}

\section{Vorbemerkungen}

Im Laufe der letzten Jahrzehnte ist auf den Spuren der Kulturanthropologie eine selbständige neue Forschungsrichtung, die sich mit der interkulturellen Kommunikation befaßt, entstanden. Im Vergleich zu den theoretischen und empirischen Überlegungen und Feststellungen der Anthropologie konzentrieren sich die Forscher unserer Zeit mehr und mehr auf die Konflikte und Lösungen, welche dann entstehen, wenn bekannte, beschriebene, aber doch unterschiedliche Kulturmodelle aufeinanderprallen. Diese Herausforderung ist aus zwei Richtungen auf die Forscher gekommen: einerseits sind die Konsequenzen der kulturellen Konfrontationen aus der Kolonialzeit der Geschichte allzu offensichtlich und gravierend, um sie nicht zu berücksichtigen, andererseits zwingen die sich globalisierende Wirtschaft und ihre Verbündeten (Internet, Börsen etc.) die Akteure immer mehr, an mehreren Schauplätzen präsent und aktiv zu sein. Das führt unvermeidlich zu drastischen Konflikten, die wiederum sehr unterschiedlich interpretiert werden können (ein Beispiel dafür stellt das vieldiskutierte Buch von S. Huntington: „The Clash of Civilisations..." dar).

Die Aufgabe, die den Forschern gestellt wurde, ist äußerst kompliziert, ja eigentlich unlösbar, denn sie umfaßt sämtliche Dimensionen der individuellen und sozialen Existenz der Menschen. Der Erfolg der Forschung hängt mehr oder weniger davon ab, wie korrekt sie die Relevanz, die Reihenfolge nach Wichtigkeit der zu untersuchenden Komponenten einschätzt. Da wir in einer globalen Zeit leben, dürfte sich eigentlich keiner mehr Fehleinschätzungen leisten, denn die Folgen würden das Ganze betreffen, die Freiheit der Partikularität des Handelns gehört zunehmend der Vergangenheit an.

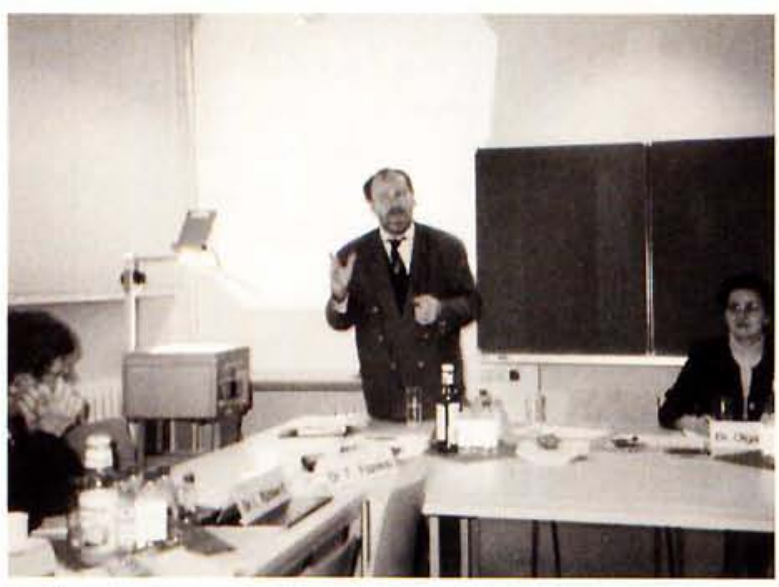

Dr. Tiborc Fazekas
Angesichts des gewaltigen Konfliktpotentials, das durch das Versagen der interkulturellen Kommunikation entstehen kann und vor unseren Augen tagtäglich auch entsteht, möchte ich auf das Dilemma, womit die Autoren der Pilzsammelbücher ständig konfrontiert werden, hinweisen: Sollen wir alles detailliert zu beschreiben versuchen, wodurch zwar der Informationswert des Buches steigt, der Leser jedoch die feinen Unterscheidungsmerkmale nicht mehr auseinanderhalten kann, seine Aufmerksamkeit leicht nachläßt und sich somit in Gefahr begibt, oder sollen wir alles auf die größten, tödlichen Gefahren konzentrieren und sagen, außer den zwei (drei, vier etc.) wirklich tödlichen Arten darf jeder sammeln, was er kann, denn er wird noch erfahren können, ob er das Richtige oder das Falsche gesammelt hat? Die Umstände verlangen von uns sehr oft sofortiges Eingreifen, ohne das wahre Ausmaß und alle Aspekte unseres Eingriffs eingeschätzt zu haben.

Auch wenn wir uns nur auf das Allernotwendigste konzentrieren, bleibt reichlich viel zu tun. Denn die „Pilze“ der interkulturellen Kommunikation sind zusätzlich auch von der jeweiligen Umgebung abhängig giftig oder genießbar. Die visuelle Welt der modernen Fernsehwerbung kann diametral entgegengesetzte Reaktionen bei verschiedenen Betrachtern hervorrufen, wohlgemeinte Gesten können tödliche Gefahren herbeirufen, durch Zunahme der Fläche der Berührungen steigen andauernd auch die Möglichkeit und die Zahl der Fehler. Und weil die menschlichen Aktionen immer Absichten vermitteln, können die gravierenden Fehlleistungen auch die ehrlichen Absichten diskreditieren, und so vielleicht überlebenswichtige Kooperation unmöglich machen.

Um all diesen Gefahren entgegenzuwirken, sind die Sozialwissenschaften wieder einmal genötigt, sich mit relevanten Phänomenen zu beschäftigen. Sie sind gleichzeitig einem zunehmend starken Druck seitens der Wirtschaft ausgeliefert, denn für die Wirtschaft bedeuten sämtliche Reibungen innerhalb der Produktion-Absatz-Kette einen Verlust, der beseitigt werden muß. Aus der klassischen Soziologie, aus der Kulturanthropologie und aus verschiedenen historischen und modernen interdisziplinären Ansätzen (wie z. B. der Imagologie) entsteht in unserer Zeit ein Konglomerat, dessen Aufgabe, wie soeben definiert, recht klar ist, bei den Methoden und Ergebnissen ist jedoch das Bild noch ziemlich verwirrend.

Ein Problem bedeutet gerade die starke Bestimmtheit der Forschung durch die Wirtschaft: Die größten Untersuchungen sind als Aufträge von Großunternehmen 
durchgeführt worden, was auch bei den besten Bemühungen zu eingeschränkten Ergebnissen geführt hat. Das bekannteste Beispiel dafür ist die vielleicht weltberühmte Arbeit von Gert Hofstede („Interkulturelle Zusammenarbeit“), die, aus einer IBM-Studie emporgewachsen, versucht, allgemeine Zusammenhänge der interkulturellen Kommunikation festzustellen. Neben klaren und wichtigen Aussagen kann man hier allerdings auch typisch lapidare Feststellungen in einem wissenschaftlichen Gewand entdecken über Tatsachen, die in einer nicht-so-formulierten Fassung eventuell besser zu verstehen sind als mit einer flachen Formulierung ( $\mathrm{z}$. B. „mentale Software“ etc.). Immerhin ist es Hofstede gelungen, die Aufmerksamkeit zu wecken für die komplizierten interpersonalen und „intersozialen“ Aspekte des menschlichen Handelns. Aber die Aufgaben der Forschung sind sowohl im Bereich der Empirie als auch bei der Methoden- und Theoriebildung noch enorm groß. Mein Artikel versucht in einem einzigen, allerdings entscheidenden und äußerst komplexen Bereich, nämlich in der Sprache, zwischen Deutschen und Ungarn vorstellbare und bestehende Probleme und Unterschiede zu erörtern. Im Rahmen einer solchen Publikation können allerdings diese Phänomene nur angedeutet, gezeigt, maximal exemplarisch dargestellt werden, ihre tiefgehenden Zusammenhänge, ihre Komplexität und ihr Umfang benötigen, um dargestellt zu werden, weit umfangreichere Formen der Aufarbeitung.

\section{Die Sprache und die Sprecher}

Die Sprache ist zwar bloß ein Feld der interkulturellen Kommunikation, aber es ist jedem klar, daß die Sprache als physiologisch-psychologisches, historisches und gesellschaftliches Produkt zugleich eine der komplexesten Phänomene des Menschen darstellt. Auch jene Kulturen, welche dem Handeln den Vorzug gewähren (im Orient), haben ihre verbalen Territorien entfaltet, und heute findet - dank der technischen Entwicklung - die Kommunikation zunehmend verbal statt, auch wenn den Computern zufolge der Mensch teilweise aus diesem Prozeß immer mehr aussteigen kann (muß, soll). Solange wir keine rein technische Form der Kommunikation zwischen Maschinen entwickelt haben, werden Menschen, wird die menschliche Komponente immer in diesem Prozeß mit dabei sein und ihn beeinflussen. Darum sind interkulturelle Studien ebenso wichtig wie die Förderung der Fähigkeit und Bereitschaft zur Empathie durch Bildung, denn das rein technische Wissen versagt auch bei perfekter Anwendung unter Menschen, die Unfähigkeit zur Interaktion fängt am häufigsten bei der fehlenden Flexibilität in Bezug auf die Menschen an. Beispiele für die Komplikationen der interkulturellen Kommunikation liefern unsere Alltagserfahrungen überall und zu jeder Zeit, auch in Mitten Europas.

Ein besonders interessantes Fallbeispiel können für Deutsche Ungarn und die Bewohner dieses Landes liefern, denn

- die Landessprachen dieser beiden Länder sind typologisch unterschiedlich (strukturelle Komponenten);
- beide Länder haben traditionelle Bindungen zueinander und zu ihren übrigen Nachbarn, aber beide sind an der Peripherie einer größeren Region - Mittelbzw. Ostmitteleuropa - und verstehen sich selbst seit Jahrhunderten als Mittler von Kulturen (historische Komponenten);

- für mehrere Jahrzehnte wurden diese Länder und ihre Kulturen durch eine der krassesten Trennerscheinungen (Eiserner Vorhang) der modernen Geschichte voneinander ferngehalten (politisch-soziologische Komponenten);

- historisch betrachtet gehören zwar die Ungarn seit mehr als tausend Jahren zum europäischen Kulturkreis, aber ihr Verhalten und auch ihre Sprache haben sehr viele Elemente ihrer „andersartigen“ orientalischen Herkunft bis heute bewahrt (psychologischkulturelle und soziale Komponenten).

Diese Faktoren reichen bereits, um eine Reihe von Mißverständnissen und Kommunikationsfehlern zu verursachen. Im Rahmen eines Vortrags kann ich allerdings nur kurz auf die letzterwähnten drei Kategorien von Komponenten eingehen, denn das Hauptanliegen meiner Ausfürungen sind die sprachlichen, die sprachbedingten Probleme. Auch bei der Behandlung der einzelnen Kategorien beschränke ich mich hauptsächlich auf die sprachlichen Manifestationen der Unterschiede.

\subsection{Die psychologisch-kulturellen und sozialen Komponenten}

Hier kann jeder, der bereits einen Ungarn getroffen hat oder Ungarn besucht hat, zahlreiche Fälle, 'mal anekdotisch, 'mal verärgert erwähnen, wo die Unterschiede sehr auffällig spürbar werden. Die traditionelle Gastfreundschaft der Ungarn gehört zu den positiv gewerteten Eigenschaften, welche genauso zu den orientalischen Merkmale der ungarischen Kultur zählen, wie das - von den Deutschen zumindest - sehr negativ bewertete Zeitverständnis der meisten Ungarn. Die Pünktlichkeit, mit der deutsche Menschen Termine wahrnehmen, ist für die „normalen“ Ungarn eine Seltenheit, bzw. die „pünktlichen“ Ungarn sind bereits assimiliert an die „westlichen“ Benehmensformen. Eine Verabredung ist für den einfachen Menschen eine Art Absichtserklärung, die er wirklich bemüht ist einzuhalten und nur unter zwingenden Umständen verletzt.

Da aber prinzipiell die menschlichen Elemente des Lebens (Edward T. Hall spricht in seinem Buch „Beyond Culture" von einer „polychronen Gesellschaft") den formalen Komponenten gegenüber Vorrang haben, kann es durchaus dazu kommen, ja es ist geradezu damit zu rechnen, daß ein Treffen mit einem Bekannten oder Kollegen etwas länger ausfällt als geplant, die Strecke zum Treffpunkt etwas mühsam bewältigt wird und der ungarische Partner grundsätzlich nach dem vereinbarten Zeitpunkt eintrifft. Diese Einstellung ist gewiß ungewöhnlich für Deutsche, die schlechte Manieren oder gar böse Absichten hinter diesem Verhalten vermuten können, und dann sind in einer solchen Situation auch richtige Konflikte zwischen den Partnern nicht mehr auszuschließen. 
Auch die geschlechtsspezifische „Empfindsamkeit“ der beiden Sprachen läßt große Unterschiede erkennen: Während im Deutschen eine zunehmend konsequente Andeutung auf das Geschlecht des Partners (Kollegin, Studentin, Antragstellerin etc.) zu beobachten ist, verhalten sich die Ungarn hier etwas verhalten. Die Möglichkeit zur Andeutung des Geschlechts ist zwar gegeben, aber die Zufügung des Elements -nö (tanárnő, doktornö etc.) hebt eine - zumindest in der Situation - sekundäre Eigenschaft des Gesprächspartners hervor, eine Frau wird sozusagen mehr als Frau und weniger als fachlich/ inhaltlich zuständige Person apostrophiert. Ein Phänomen, was geradezu im Gegensatz zu dem steht, was man in Deutschland mit der konsequenten Unterscheidung der Formen, als Gleichberechtigung der Geschlechter in den Umgangsformen, erreichen möchte.

Die Anredeformen sind ebenso problematisch, wenigstens aber „nachvollziehbar“. Für die Männer besteht die Möglichkeit, sie als „X úr“ (Herr X) anzureden. Die Probleme bei der Anrede von Frauen sind sehr komplex. Einerseits verfügt das Ungarische im Augenblick über keine vergleichbare Lösung wie das deutsche „Frau Y“. Ein Wort in diesem Kontext für "Frau“ existiert nicht. Man kann einen Versuch mit „Y asszony“ wagen, doch das ungarische Wort „asszony“ ist anders als bloß „Frau“, es ist historisch zugleich auch als „Weib“ zu verstehen, was im Ungarischen allerdings nicht so negativ konnotiert ist wie im Deutschen, im Gegenteil, das eigentliche Problem besteht gerade darin, daß die Benutzung des mit Anerkennung/Schätzung verbundenen Wortes „asszony“ im Ungarischen für Frauen nicht so neutral ist wie „úr“ bei den Herren.

Man kann massenhaft interessante Fälle erwähnen, wo der psychologisch-kulturelle Unterschied auch in der Sprache krass ins Auge springt, aber hier können nur Beispiele als Illustrationen gezeigt werden. So muß man sich intensiv mit den komplizierten Formen des Begrüßens und des miteinander Umgehens im Ungarischen beschäftigen, ein falscher Gruß, eine verwechselte Anrede kann vieles zerstören.

Kezicsókolom /Küsse die Hand/, Jó napot/Guten Tag/, Adj' Isten /Grüß Gott/, Üdvözlöm /Ich grüße Sie/, Szervusz /lch griisse Dich/, Szia /Hallo und Tschïß/ sowie Heló /ebenfalls Hallo und Tschüß/ gelten als Visitenkarten und Rangordnungs- oder Verhältnisbekenntnisse, daher ist ihr Gebrauch sehr pingelig bestimmt.

Ähnliche Regeln betreffen das Zeitgefühl: Die Ungarn mit ihren orientalischen Verhaltensstrukturen haben ein anderes Verhältnis zur Zeit als die Deutschen. Dieser Umstand führt oft zu unangenehmen Mißverständnissen und Ärger, wobei man nur über diese Tatsache informiert sein sollte. Anderswo sind wiederum die Ungarn strenger: Nur bis etwa 8 Uhr morgens soll man mit Jó reggelt /Guten Morgen/ begrüßen, insbesondere in Dörfern, denn dieser Gruß gilt in den späteren Stunden eher als spöttische Mahnung für Spätaufsteher.

Die komplizierte Hierarchie der Anredeformen ist ebenso historisch entstanden wie die Vielfalt in der Regelung des Duzens. Obwohl man behaupten kann, daß die Ungarn und ihre Kultur das Duzen gerne und als natürliche Form der Kontakthaltung praktizieren, sind die Spuren der höfischen, feudalen Kultur mit ihren strengen, etikettierten Umgansformen bis in unsere Tage hinein zu spüren. Wer, wem und in welcher Situation das Duzen anbieten darf, wieviele Stufen zwischen dem „gleichberechtigten“ Duzen und den Übergansformen vom Siezen zum Duzen (meistens weiterhin um ein gewisses Ansehen des Gesprächpartners anzudeuten) es gibt, bleibt für Fremde auch nach längerem Aufenthalt im Lande ein schwer zu lösendes Rätsel.

Die Orientierung wird auch dadurch etwas erschwert, daß die Einstellung der Ungarn der Sprache (sowohl ihrer Muttersprache als auch fremden Sprachen) gegenüber stark von der Einstellung der Deutschen abweicht. Das wichtigste Moment in diesem Zusammenhang ist die Verspieltheit, daß man die Sprache nicht als „Obrigkeit“, als Träger einer gesellschaftlichen Hierarchie oder Norm ansieht, sondern vielmehr als eine Herausforderung, um die eigenen geistigen Fähigkeiten zu artikulieren. Verdrehungen, Wortwitze, Wortschöpfungen und verbale Anspielungen gehören zu den normalen Begleiterscheinungen der Kommunikation, was für Fremde beim Verfolgen einer Unterhaltung unter Ungarn wieder sehr große Schwierigkeiten bereiten kann.

Man muß auch mit erheblichen psychologischen Unterschieden sowohl im individuellen als auch im sozialen Kontext rechnen. Wenn darauf hingewiesen wird, daß man in Ungarn viele Klagen hört, dann mag das an der tatsächlich vorhandenen Neigung zum Selbstmitleid liegen, äußerte sich jedoch jemand anderer kritisch über das Land und die Leute, würden sich die betroffenen Ungarn bestimmt wieder mehr verteidigend für ihre Heimat einsetzen. Das Recht zu nörgeln wird meistens sich selbst vorbehalten.

Auch die Einstellung der Individuen zu einer durchzuführenden Aufgabe weicht von dem üblichen west-mitteleuropäischen Muster ab. Hier sind die sprachlichen Mittel zur Überzeugung der Betroffenen besonders wichtig, denn ein blinder Gehorsam bei der Durchführung der hierarchisch verordneten Befehle kann kaum erwartet werden. Man kann sich reichlich romantische Fehlvorstellungen ausmahlen warum, aber die meisten Ungarn ertragen nur den Zwang, den sie sich selbst auferlegt haben. Sie benötigen also zunächst eine „zielorientierte" Informationsstruktur, dann brauchen sie es, wenigstens das subjektive Gefühl zu haben, daß sie selbst das Ziel und den Weg dazu gefunden haben, und wenn wir so weit gekommen sind, kann man mit Sicherheit damit rechnen, daß die Beteiligten aktiv und kreativ zur Aufgabe stehen, sehr oft mit der Folge, daß die vorgestellte Durchfuihrung schneller, leichter und besser klappt, weil die Menschen auch während der Realisierung weiter über ihr Vorhaben nachdenken und ihre umfangreichen Erfahrungen immer wieder einsetzen. Darum ist es prinzipiell effizienter, wenn man nicht mit den formalen Kriterien (Bildungsstand, Titeln, Weisungsrecht etc.) die Zusammenarbeit forciert, sondern den Unterschied zwischen den Beteiligten klein zu hal- 
ten bemüht ist. Autorität, welche nicht von unten, nicht auf Grund von Alltagserfahrungen der Mitarbeitenden entstanden ist, animiert die Menschen nämlich mehr zum Widerstand. Vergleichbare Verhaltensformen wurden - allerdings in Bezug auf andere Kulturen - auch von Geert Hofstede in seinem Buch „Uncommon Sense about Organizations" beschrieben. Natürlich benötigt dieser Umstand die Beherrschung eines außerordentlich breiten Registers von sprachlich-kulturellen Mustern. Das stellt zugleich eine der schwierigsten Aufgaben des Fremdsprachenunterrichts dar, denn bei der üblichen Vermittlung von Sprachen werden die Registerunterschiede, die soziologischen und psychologischen Faktoren und Hintergründe höchstens erwähnt, die Rahmenbedingungen des Unterrichtes lassen normalerweise ihre Einübung und Erlernen nicht zu.

Die Dynamik des Redens und des Schweigens hat auch ihre eigene Gesetze. Wer mit wem über was und wie spricht, ist auch eine sehr komplexe Frage der soziokulturellen Komponenten. Ein in einer kommunikativen Situation auftretendes Schweigen ist, wie in den meisten europäischen Kulturen, mit negativen Aspekten belastet, wobei es ein sehr „beredtes“ sein kann. Die Auflösung einer solchen Kommunikationssperre ist sprachlich keine leichte Aufgabe, aber man muß alles versuchen, sie $\mathrm{zu}$ beseitigen. Die in vielen Statistiken und traditionell hervorgehobene hohe Selbstmordrate unter den Ungarn ist gewiß eine Realität mit vielen Facetten. Das Vereinsamen, das In-sich-kehren, die Aggression gegen sich selbst manifestieren sich sehr oft, ehe sie zu Taten führen, in einer Verweigerung der Kommunikation.

Dagegen wird man immer wieder überrascht, mit welcher Offenheit Themen (z. B. die finanzielle Lage, das Einkommen) angesprochen werden, die in anderen Kulturkreisen als Tabuthemen zu den persönlichsten „Geheimnissen“ gehören. Das überschreiten von solchen kulturell geerbten persönlichen „Hoheitsgrenzen“ geschieht sehr oft unter Einwirkung von Alkohol, wozu es in Ungarn sehr viele Anlässe gibt und gefunden werden. Alkoholgenuß mit Maß, eine Situation mit lockerer Atmosphäre, das gemeinsame Kaffeetrinken, ein mit kulturellen Zeremonien praktisch geregeltes Umfeld hilft aber erfahrungsgemäß sehr oft bei der Überbrückung der vorhandenen sprachlichen Hindernisse.

\subsection{Die politisch-soziologischen Komponenten}

Die ungarische Gesellschaft hat während der vergangenen hundert Jahre tiefgreifende Erschütterungen erlebt. Die Spuren der mittel- und osteuropäischen „Verspätung“, wonach die feudalen gesellschaftlichen Verhältnisse, ein nur beschränkt entfaltetes Bürgertum, krasse Gegensätze in den politisch-materiellen Gegebenheiten das Geschehen bis zum Zweiten Weltkrieg bestimmt haben, wurden nach dem Krieg für vierzig Jahre von einem neuen Modell abgelöst. Gemeinsam war an diesen Modellen, daß sie stark von außen bestimmt und auferlegt waren. Die „Modernisierung“ der Gesellschaft fand in dieser Zeit von hundert Jahren - im übrigen nicht nur in Ungarn - faktisch so statt, daß anstelle der orga- nischen, inneren Entwicklungslinien immer mehr fremde und bezwingende Faktoren zu wirken begonnen haben. Infolgedessen stehen die Menschen in Ungarn vielfach skeptisch der Modernisierung gegenuiber (was sich je nach Gemüt vom gesunden Mißtrauen bis hin zur politisch extremen Xenophobie manifestieren kann). Ihre Attitüde, ihr individuelles Verhalten ist trotz der gravierenden Unterschiede durchaus vergleichbar mit dem, was man bei den Menschen im Laufe der Eingliederung der neuen Länder in die Bundesrepublik Deutschland beobachten kann. Die tagtägliche Konfrontation mit dem Urteil, daß das bis dahin geltende und erlebte Lebensund Gesellschaftsmodell (was natürlich zur gleichen Zeit die Menschen geprägt hat und von den Menschen geprägt wurde) nur Ablehnung erfährt, verunsichert, vernichtet den Rest des Selbstvertrauens, fuihrt psychologisch wie sozial zu gravierenden Spannungen.

\subsection{Die historischen Komponenten}

Die Klischees und Vorurteile sind wichtige Komponenten der menschlichen Kultur, die durch die Kulturgeschichte tradiert und immer wieder belebt werden. Eine frühe Aufzeichnung im westlichen Europa über die damals gefürchteten Ungarn stützt sich auf alte sprachliche Überlieferungsformeln in Bezug auf „barbarische“ (=e twa nicht zum eigenen Kulturkreis gehörende) Völker, und beschreibt sie wie folgt: „Sie seien klein gewachsen, kahlgeschoren, hätten abstoßende Gesichtszüge, tiefliegende Augen, ihre Sprache und Geschrei sei abscheulich (Heraushebung von mir - T. F.), sie seien eher Tiere als Menschen, äßen rohes Fleisch, tränken Blut... Ihrer Veranlagung nach seien die Ungarn schlau, hinterlistig, meineidig und vertragsbrüchig. Das bezeugt ihre ungewöhnliche Kriegstaktik: Sie stellen sich dem Feind nicht im offenen Kampf, sie stellen Fallen, imitieren die Flucht, wenden sich aber plötzlich um und vernichten die in Unordnung geratenen Verfolger."1

Sicherlich hat sich in der Zwischenzeit vieles verändert, und die obigen Klischees werden etwas umgestaltet auf andere Kulturen und Völker übertragen, aber die Sprache der Ungarn wird auch heute noch immer wieder als eine von den „nicht-erlernbaren“ dargestellt. Infolge dieses Rufes neigen die meisten Fremden dazu, zu erwarten, daß ihre ungarischen Partner fremde Sprachen sprechen. Ein „sprachbedingtes“ Hindernis besteht eben in dem nicht ausreichenden Vorhandensein dieser Sprachkenntnisse: Nach einer Statistik haben 1990 in Ungarn aus der gesamten Bevölkerung 4,37\% Deutsch, 2,21\% Englisch, 1,52\% Russisch als „bekannte-beherrschte“ Fremdsprache angegeben. ${ }^{2}$ Auch das führt zu leichten Irritationen: Während man sich in der breiten Welt gut mit den wichtigsten indogermanischen Sprachen (Englisch, Französisch, Deutsch, Russisch etc.) durchsetzen kann, helfen diese Kenntnisse in Ungarn nur sehr beschränkt weiter. Die gelegentlich vorhandene Bereitschaft, sich auch in geschäftlichen Beziehungen auf das Ungarische zu stuitzen, verlangt entweder einen Vorrat von ständig einsatzbereiten Übersetzern oder aber, daß die Fremden Ungarisch lernen. Auch wenn das noch überhaupt nicht als typisch bezeichnet werden kann, 
gibt es zunehmend viele ermutigende Beispiele fuir die Bereitschaft dazu. Allerdings müßten die ganze Unterrichtsmethodik, der Unterrichtsinhalt, und auch die Unterrichtsbüicher und Lehrer des Ungarischen völlig neu konzipiert und eingesetzt werden.

\subsection{Die strukturellen Komponenten}

Will jemand die Sprache der Ungarn erlernen, muß er eine ganze Reihe von Abweichungen von den üblichen Fremdsprachen im Laufe des Studiums bewältigen. Ungarisch, als Mitglied der finnisch-ugrischen Sprachfamilie, weicht in seiner Struktur - trotz einer inzwischen ein Jahrtausend lang gewordenen Nachbarschaft - von den indogermanischen Sprachen gravierend ab. Mit etwas Übertreibung kann man behaupten, das fuir westeuropäische und somit deutsche Muttersprachler Ungarisch zu lernen ein vergleichbares Unternehmen darstellt, als wenn sie außereuropäische Sprachen (Japanisch, Arabisch, Kechua etc.) zu lernen beginnen würden, auch wenn die Ungarn selbst oder die Finnen auf dem Kontinent Europa leben. Durch ihre lange Wanderung haben die Ungarn etwas ganz Fremdes, nämlich ihre Sprache in die Mitte Europas gebracht. Zahlreiche Einfluisse haben im Laufe der Zeit diese fremde Sprache zwar beeinflußt, zum Teil an die indogermanischen Sprachen angenähert, aber die Grundstrukturen sind weiterhin deutlich anders geblieben. Im Folgenden möchte ich nur einige dieser Besonderheiten, die auch bei der Kommunikation und Verständigung eine bedeutende Rolle spielen, vorstellen.

\section{A) Das Schriftbild und die Aussprache der Wörter}

Obwohl das Ungarische mit lateinischen Buchstaben geschrieben wird, ist es für Fremde nicht einfach, schreiben und lesen zu lernen. Den Grund für diese Schwierigkeiten stellen die 39 Laute des Ungarischen dar, die mit den 25 Buchstaben des lateinischen Alphabets schriftlich widergegeben werden. Diese Diskrepanz wird zwar recht konsequent mit Nebenzeichen (bei Vokalen wie á, é, í, ó, ö, ú, ü ) und Graphemkombinationen (bei Konsonanten cs, sz, zs, bzw. gy, ly, ny, ty) überbrückt, das phonematorische Prinzip (jedes Graphem nur ein Laut und jeder Laut nur ein Graphem) wird weitgehend eingehalten, trotzdem bedarf es einiger Zeit, bis die gewïnschte Lesesicherheit entsteht.

Die Regeln der Aussprache bringen jedoch mehr Unsicherheiten. Als allererstes Problem muß man den Unterschied in der Artikulation hervorheben: das Ungarische duldet keinerlei Reduktion der Vokale, jedes Graphem zeigt einen voll auszusprechenden Laut. Die ungarische Aussprache im Deutschen wie [jeden, gehen] etc. ist allgemein als charakteristisch bekannt, es klingt jedoch vergleichbar merkwürdig, wenn in bestimmter Lautumgebung die deutschen Muttersprachler automatisch, entsprechend ihrer Muttersprache die Wörter so artikulieren: [k'özl] statt [közel]. Die typische und regelmäßige Reduktion der Laute im Deutschen führt zu Unsicherheiten bei der Aussprache von langen Vokale und Konsonanten, im allgemenien geraten sie ständig zu kurz.
Wenn es einem gelingt, sich die gesetzmäßige Konsonantenverhärtung des Deutschen abzugewöhnen, dann bleiben nur die komplizierten Assimilationsgesetze der ungarischen Aussprache zu bewältigen. Selbstverständlich kommt noch das völlig fremde Phänomen, die Vokalharmonie (palato-velare und labial-illabiale Versionen), die - allerdings bisher wissenschaftlich noch nicht erforscht - auch von gewissen Komponenten einer Art „Konsonantenharmonie" (spezifisch bei den Möglichkeiten der einzelnen Laute in ihrer Kombinatorik) ergänzt werden. Alles in allem eine gewaltige Menge an Neuigkeiten, die allesamt gleich zu Beginn des Studiums erklärt werden müssen, sonst ist keine Anwendung des Erlernten in der Praxis möglich.

Auch eine weiterfuihrende, morphonematische Erscheinung gehört zu den problembereitenden Elementen des Erlernens: Die Frage der sogenannten Bindevokale. Entsprechend der soeben angesprochenen Regeln der Kombinatorik der Konsonanten (deren Häufung das Ungarische im allgemeinen zu meiden bemüht ist), muß die flexionsbedingte Kollision von Konsonanten durch „Dazwischenschieben " von bestimmten Vokalen vermieden werden. Wären diese „Bindevokale“ allerdings wirklich nur Bindevokale, wären sie nur dem Gesetz der Vokalharmonie unterworfen, reichten maximal drei (ein palatal-illabial z. B. -e, ein palatal-labial z. B. -ö und ein velar z. B. -o) von ihnen aus. Da diese drei allerdings im heutigen Ungarisch nicht ausreichen, wird man gleich mit der vielleicht gravierendsten „Fremdheit“, mit der ständigen Präsenz der Geschichte der Sprache in den Formen der Gegenwartssprache des Ungarischen konfrontiert. Dieser Umstand ist zum Teil verantwortlich für die gefürchtete Formvielfalt und den oft nachgesagten Reichtum an Unregelmäßigkeiten des Ungarischen. In Wirklichkeit sind dies am seltensten Unregelmäßigkeiten, bloß die Sprache hat im Laufe ihrer Geschichte mehrere Phasen der Entwicklung durchgemacht, die Prozesse haben sich einerseits selten hundertprozentig durchgesetzt, andererseits wurden auch fruiher praktizierte Formen nicht restlos verdrängt, einige Wörter richten sich weiterhin nach „untrendmäßigen“ Regeln. Es gibt zahlreiche Parallelitäten bei der Lösung zur Mitteilung desselben Inhaltes, und man muß - wieder eine kleine Unannehmlichkeit des Ungarischen - gleichzeitig in mehreren Systemen denken, sie parat haben. Bei der Herstellung größerer sprachlicher Einheiten erlebt man richtig die strukturell-systematische Vielfalt der Sprache, zur größeren Freude vor allem der Transformationsgrammatik.

\section{B) Die Schwierigkeiten mit einer reichen Morphologie}

Diese gerade angedeuteten größeren Einheiten entstehen im Ungarischen mit einem weniger fremden Mittel, mit der Flexion der Wörter, so, wie das die agglutinierende Natur der Sprache verlangt. Die Agglutination ist recht einfach zu verstehen: Prinzipiell wird jede sprachliche Funktion mit einem (und nur mit einem) Zeichen - in diesem Fall mit Endungen, Suffixen - erfüllt. Die ungarischen Wörter „wachsen nach hinten“, jede Information über Modus-Tempus-Person beim Verb und Numerus-Kasus-etc. beim Substantiv wird in einer bestimm- 
ten Reihenfolge an den Wortstamm angehängt. Diese synthetische Morphologie des Ungarischen ähnelt danach einem recht komplizierten Dominospiel. Deutschungarische Kontraste sind hier auch gravierend. Während im Deutschen nach analytischem Prinzip die Komponenten kombiniert werden können, wie z. B.

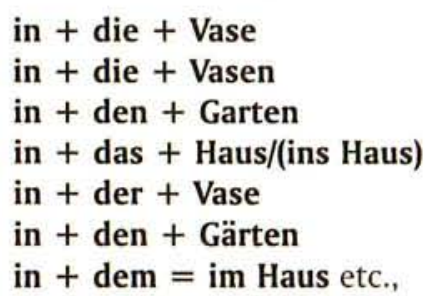

wird im Ungarischen einer synthetisierende Kombinatorik gefolgt. Diese ist nicht weniger formreich und aufregend, nur sie funktioniert völlig anders:

Nomen

Verb

kert(1)-e(2)-k(3)-ben(4) kér(1)-het(5)-t(6)-e(2)-tek(7)

(1) Wortstamm

(2) „Bindevokal“

(3) Pluralzeichen (absolute Flexion)

(4) Lokalendung (für Wo + drin), als vermeintlicher „Kasus" (denn es ist im deutschen bereits die Kombination von einem Kasus und einer Präposition: in + Dativ) wird sie in der Fachliteratur als Inessiv bezeichnet

(5) Potentialzeichen (dt. 'können')

(6) Zeichen der Vergangenheit (nur eine): Präteritum

(7) Personalendung: 2. Plural

Während man im Deutschen also die Information „linear einengen muß“ (nach in gibt es oben noch 8 Optionen, nach in + die und in + den nur noch jeweils 2-2, nach in + das, in + der und in + dem bleiben jeweils nur eine), schließt man im Ungarischen mit den monofunktionalen Suffixen mit einem jeden Dominostein eine Domäne vollständig ab: Wenn das absolute Pluralzeichen -k (3) auftaucht, kann es kein Possessiv mehr geben, wenn das Inessivzeichen -ben (4) vorkommt, können keine weiteren Lokal- oder sonstigen Kasusendungen mehr angehängt werden, diese Endungen stellen immer das Schlußlicht einer Form dar, usw.

Dieses Dominoprinzip des Ungarischen kann dann wirklich zu verblüffend logischen Konsequenzen führen, aber diese klare Logik kann auch fremd und somit erschwerend wirken. Wenn die obige Personalendung -tek (7) für die 2. Person Plural steht, kann man sich auch einen Bezug zwischen dem obigen Substantiv kert (1) und dieser Personalendung vorstellen, selbstverständlich, da Konsonanten aufeinandertreffen würden, unter Anwendung des „Bindevokals“-e (2). Die neue Form sieht dann so aus:

\section{kert(1)-e(2)-tek(7)}

etwa Garten + ihr

und bedeutet tatsächlich: euer Garten

Will ich noch einen Lokalkasus zufuigen wie z. B. -ben (4) von oben, darf ich das auch tun, am Ende, diesmal aller-

dings geht es auch ohne einen weiteren „Bindevokal“:

$$
\text { kert(1)-e(2)-tek(7)-ben(4) }
$$

etwa Garten + ihr + in und es bedeutet: in eurem Garten

Nimmt man jedoch die „Bedeutung“ 'in etwas drin' (4) auf Personen bezogen wahr, kann auch - korrekt - die Form entstehen:

$$
\begin{aligned}
& \text { benn(4)-e(2)-tek(7) } \\
& \text { etwa drinnen }+\mathrm{ihr}
\end{aligned}
$$

und es bedeutet: in euch

Diese Art der Kombinationen von morphologischen Elementen ist im Falle der deutschen Sprache unvorstellbar, denn dort ist die Reihenfolge der Komponenten auch festgelegt.

Die tatsächliche Lage ist allerdings noch verblüffender. Man kann feststellen, daß es keine zwei Sprachen unter den bekannteren gibt, die in ihrem Aufbau so komplementär und in ihrer Morphologie so einander diametral gegenüberstehen, wie gerade Deutsch und Ungarisch. Dieser Umstand verlangt von den Muttersprachlern beider Sprachen beim Erlernen der jeweils anderen Sprache eine enorme Umstellung des Denkens. In Anlehnung an das oben gezeigte Beispiel kann man die Komplexität der Vorgänge in der Morphologie im Deutschen und im Ungarischen bei den folgenden Formen miteinander vergleichen:

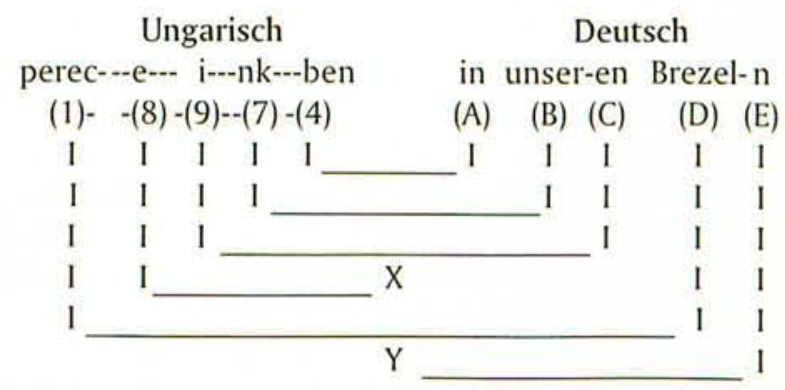

In Elementen:

$$
\begin{array}{cc}
[a)+b)+c)+d)+e)] & {[a /]+[b /+c /]+[d /+e /]} \\
\text { ein Wort } & \text { drei Wörter }
\end{array}
$$

Beide Formen werden aus fünf Komponenten aufgebaut [Ungarisch: a) bis e) bzw. Deutsch: a/ bis e/], wovon zwei (pro Sprache nur eine), nämlich $\mathrm{X}$ und $\mathrm{Y}$ etwas Spezielles, in der anderen Sprache in dieser Form nicht Vorhandenes, darstellen.

Die Reihenfolge der einzelnen Elemente ist allerdings entscheidend unterschiedlich. Ein ungarischer Muttersprachler muß die aus seiner Sicht logische Reihe (a)e)] nach der deutschen Folge konstruieren, die, mit den Symbolen der eigenen (ungarischen) Struktur angezeigt, so aussehen muß:

$$
[e)]+[d /+c /]+[a /+Y]
$$

Das eigene Element b/ fehlt in dieser Konstruktion, dafür taucht das in der eigenen Sprache völlig unbekannte Phänomen $Y$ zwingend auf. 
Für einen deutschen Muttersprachler ist dieselbe Aufgabe genauso schwer, denn er muß die in der Muttersprache übliche Reihenfolge der Elemente [a/-e/] durch die Folge der anderen Sprache ersetzen, die, mit den Symbolen der eigenen Sprache ausgedrüickt so aussieht:

$$
[d /+X+c /+b /+a /]
$$

Hier entfällt das Element e/, dafür muß jedoch das unbekannte Element $\mathrm{X}$ eingesetzt werden.

\section{C) Die Qualen der Lexik}

Die Erfolge des Fremdsprachenunterrichts in der Schule hängen auch damit zusammen, daß die dort unterrichteten Sprachen typologisch einander meist nahe verwandt sind. Diese Tatsache spielt eine entscheidende Rolle bei der Aneignung des „fremden“ Wortschatzes, denn der Lernende kann mit geringfiigigen Lautanpassungen die fremden Wörter aus den Wörtern seiner eigener Muttersprache „selbst herstellen“. Dank der einstigen kulturvermittelnden Rolle des Lateins darf man die deutsche Konstitution neben Grundgesetz als ebenbürtig ansehen, wodurch eine leichte Brücke zum englischen constitution, zum französischen constitution, ja sogar zum russischen конституция hergestellt wird.

Bei der Überschreitung der Grenzen der sprachlichen Verwandtschaft, und so auch im Falle des Ungarischlernens, müssen wir leider auf diesen sehr angenehmen Mechanismus verzichten. Plötzlich spielen die individuellen Fähigkeiten der Memorie unerwartet direkt mit im Lernprozeß, der Lernende wird mit absolut unbekannten Wortformen überschüttet, denn er muß für das oben erwähnte Beispiel das ungarische Wort alkotmány lernen. Man könnte zwar auch hier auf die lateinische Tradition zurückgreifen (in Ungarn war Latein noch bis Ende des 18. Jahrhunderts Amtssprache), aber der Gebrauch des heute archaisch klingenden Wortes konstitúció würde nur in gebildeten Ohren Gehör finden.

Ähnlich wenig Abhilfe ist von den im Laufe der Jahrhunderte ins Ungarische aufgenommenen deutschen Lehnwörtern zu erwarten. Einerseits war dieser Einfluß des Deutschen stark regional geprägt, hauptsächlich süddeutsche und österreichische Wörter gelangten ins Ungarische, sie sind im gesamten deutschen Sprachraum nicht unbedingt bekannt, sie sind also auch nicht hilfreich: Kipferl ist kifli, Krumbeer ist krumpli, Hundsfutt ist huncut geworden etc. Andererseits haben sich diese Entlehnungen dann im Ungarischen oft erheblich verändert, und dieser Prozeß der Anpassung kann sie heute etwas fremd vorkommen lassen: Wurst ist zum virsli, Bäcker zum pék, Pranger zum pellengér geworden etc. Der Gebrauch des international verbreiteten lateinischen Wortschatzes läßt die Sprache allzu archaisch klingen, die neueren, international kursierenden Wörter ermöglichen nur eine inhaltlich stark eingeschränkte Kommunikation, also bleibt nichts anderes übrig als die strenge und andauernde, memorierende Einprägung des ungarischen Wortschatzes.

\section{D) Freier Satzbau mit strengen Regeln}

Auch in der Syntax zeigt die ungarische Sprache ihre Eigenartigkeiten (wie das Fehlen einer Fragesatzwortfolge), welche einerseits aus streng befolgten, jedoch anders wirkenden Regeln bestehen. Andererseits stellt das Syntagma das entscheidende Element des Satzes dar: Syntagmen werden nach festen Regeln aufgebaut, ihre Reihenfolge innerhalb des Satzes ist aber intentionsabhängig, kann „nach Belieben“ verändert werden. Dadurch entsteht das Gefühl, daß die Syntax eben sehr locker wäre, sie befolgt aber lediglich auf der „vorsyntaktischen" Ebene strenge Regeln.

Ein anderes, gravierendes Problem stellt die „Objektlastigkeit" des ungarischen Satzes dar: Die Formen des Verbs werden von der „Konkretheit“, von der „Bestimmtheit" des Objekts regiert. Diese Besonderheit führt nicht nur zu einer Vielzahl von grammatischen Formen, welche zu erlernen viel Zeit kostet, sie verlangt auch anders geartete Denkprozesse beim Herstellen eines ungarischen Satzes. László Keresztes hat diese Probleme beim ungarischen Satzbau sehr plastisch geschildert: „Im Englischen kann man aus der Grundform der Wörter im Wörterbuch leicht folgenden Satz zusammenstellen: I like coffee, und dementsprechend: I like tea, beer, milk etc. Solche und ähnliche Konstruktionen kann man schon in der ersten Unterrichtsstunde leicht produzieren. (...) Die (ungarische - T. F.) Form szeretem a kávét, teát, sört, tejet setzt (aber - T. F.) noch mehr morphologische und syntaktische Kenntnisse voraus (...). Man muß den Unterschied zwischen der indeterminativen und (der - T. F.) determinativen Konjugation und die Regeln ihrer Anwendung lernen, in Zusammenhang damit den Gebrauch des bestimmten Artikels (der sich auch in diesem Fall grundlegend von dem im Englischen unterscheidet), weiter die morphologischen Probleme der Verbindung des Akkusativsuffixes mit dem Stamm: Die Dehnung a : á am Stammauslaut und die Alternanten des Akkusativsuffixes bei konsonantischen Stämmen." 3

Der Weg zum Bau eines inhaltlich einfachen ungarischen Satzes ist auf der Ebene der Morphologie mit so vielen „Neuigkeiten" belastet, daß die freie und aktive Herstellung von Aussagen im Lernprozeß erst etwas später möglich wird, was die Motivation, das Gefühl des Vorankommens, beim Lernenden durchaus spürbar belasten kann.

\section{E) Eine fremde Kultur im Spiegel einer fremden Sprache}

In der subjektiv empfundenen Fremdheit einer Sprache kann man die Eigenartigkeiten der fremden und der eigenen Sprache gleichermaßen deutlich erkennen. Sprachen sind sozusagen die in historischem Ausmaß geprägten verbalen Abdrücke der sie benutzenden Menschen und Kulturen. So steht auch die ungarische Sprache vielfach als Vertreter der ungarischen Denk- und Verhaltensmuster vor dem Lerner. Diese Eigenartigkeiten können - zum Glück - die sonst oft mit schwerem und komplexem Lernstoff belasteten Unterrichtsstunden etwas lockern und schwächer gewordenen Mut und ebensolche Motivation wiederbeleben, denn die Menschen selbst erscheinen hinter den sprachlichen Formen und Normen. 
Im allgemeinen hilft die plastische, bildhafte Sichtweise des Ungarischen dem Lernprozeß sehr. Die früher bereits als Hindernis erwähnte Sprachgeschichte bringt hier verbliiffend positive Beispiele. Das heutige ungarische Wort für Stadt ist eine Ableitung, bestehend aus den Elementen vár /Burg/ + -o /Bindevokal/ + -s /Nominalableitung: Nomen possessoris/, also „eine Burg besitzende (Siedlung)“. Das ungarische Wort város trägt in seiner Form sozusagen den ganzen kulturhistorischen Inhalt des Begriffs „Stadt“ in sich.

Vergleichbare und verspielte Beispiele gibt es zum Glüick des Lehrenden auch aus dem gegenwärtigen Sprachgebrauch immer noch massenhaft, wie z. B. die Bezeichnung des Begriffs „Puzzle“, was in der Umgangssprache heute als gumihangya /etwa Gummiameise/ bezeichnet wird, gleichzeitig auf die verwirrende Masse und Ähnlichkeit der Elemente /Ameisen/, als auch auf die Folgen des übermäßigen Genusses /Gummi -Zelle-/ hinweisend.

\section{Schlußbemerkungen}

Die kulturgeprägten, sprachlich überlieferten Unterschiede zwischen Deutschen und Ungarn sind trotz jahrhundertelanger Nachbarschaft vorhanden. Ihre gezielte Erforschung, ihre Anwendung im Sprachunterricht, ihre Berücksichtigung bei der Vorbereitung von Verhandlungen im geschäftlichen wie im politischen Leben stellt eine unmittelbare und unausweichbare Aufgabe für uns dar.

Hinter einer andersartig funktionierenden Sprache verbirgt sich nämlich eine andere Betrachtungsweise der Menschen, und mit Hilfe dieser anderen Betrachtungsweise können wir über unsere gemeinsame und eine Welt andere Informationen, andere Erkenntnisse bekommen. Die Mißverständnisse, die sprachlichen Konfrontationen gewähren uns somit eine Chance, die Fehlbarkeit unserer Einstellung, unseres Wissens zu erkennen und sie somit zu korrigieren. Letztlich helfen diese im Alltag zumeist bloß als peinlich oder als störend empfundenen Kleinigkeiten, große und wichtige Veränderungen in uns und um uns herum einzuleiten. Vorausgesetzt, daß wir die subjetive Bereitschaft und die objektive Möglichkeit dazu noch nicht endgültig verloren haben.

\section{Fußnoten}

1 Seewann, Gerhard: „Mégis huncut a német.” Zum Ungarn- und Deutschlandbild in der Zwischenkriegszeit. In: Das Ungarnbild in Deutschland. München, 1996. S. 63-73.

2 Kiss, Jenö: Társadalom és nyelvhasználat/Gesellschaft und Sprachgebrauch/. Budapest, 1995. Tabelle 17 auf S. 174 .

3 László Keresztes: Die Probleme der Ungarisch lernenden Finnen. Hungarologische Beiträge $\mathrm{Nr}$. 2. Jyväskylä 1994. S. $127-128$.

\section{Literatur}

Hall, Edward T.: Beyond Culture. London 1976, Doubleday Anchor Books.

Hofstede, Geert: Interkulturelle Zusammenarbeit. Wiesbaden 1993, Gabler Verlag.

Hofstede, Geert: Uncommon Sense about Organizations. Cases, Studies, and Field Observations. London, New Delhi 1994, Thousand Oaks.

Huntington, Samuel: The Clash of Civilisations and the Remaking of World Order. New York, N. Y. 1996, Simon \& Schuster.

\section{Verfasser}

\section{Dr. Tiborc Fazekas}

Finnisch-Ugrisches Seminar der Universität Hamburg Bogenallee 11

20144 Hamburg

Tel. +49 (0) 40 - 41232788 